\title{
RDUS
}

Revue de DROIT UNIVERSITÉ DE SHERBROOKE

Titre : $\quad$ QUEL EST L'AVENIR DU GRIEF PATRONAL?

Auteur(s): $\quad$ Carole SÉNÉCHAL

Revue : $\quad$ RDUS, 2013, volume 43, numéro 3

Pages: $\quad 793-826$

ISSN : $\quad 0317-9656$

Éditeur : $\quad$ Université de Sherbrooke. Faculté de droit.

URI : $\quad$ http://hdl.handle.net/11143/10219

DOI : https://doi.org/10.17118/11143/10219 
Page vide laissée intentionnellement. 


\title{
ARTICLE
}

\section{QUEL EST L'AVENIR DU GRIEF PATRONAL?}

\author{
par Carole SÉNÉCHAL*
}

Le développement du syndicalisme a en quelque sorte bridé l'arbitraire de l'employeur en milieu de travail en assumant la défense des intérêts de certains groupes de salariés par l'entremise de la négociation périodique des conditions de travail. Désormais, le syndicat majoritaire accrédité et l'employeur sont tenus de négocier de bonne foi une convention collective définissant les droits et obligations de chacun. Devant cette dissolution de l'autorité de la direction, on assiste peu à peu à l'émergence du phénomène relativement récent du grief patronal en vue de dénoncer le non-respect par la partie syndicale des dispositions de la convention collective. Force est toutefois de constater que les sentences arbitrales disposant des griefs patronaux demeurent rarissimes, ce qui n'est probablement pas étranger à la baisse du taux de syndicalisation.

The development of unionization has kept the arbitrary power of employers in the workplace in check by defending the interests of certain paid workers through the periodic negotiation of working conditions. As a result, the majority accredited union and the employer are bound to negotiate a collective agreement in good faith which defines the rights and obligations of each. Faced with this dissolution of management's authority, we have come to witness little by little the emergence of the recent phenomenon of employer grievance in an attempt to denounce the union's non-compliance with the articles of the collective agreement. One can see that the arbitral rewards from employer grievances remain rare, which is possibly not unrelated to a decrease in the rates of unionization.

\footnotetext{
* . Professeure à la Faculté d'éducation de l'Université d'Ottawa.
} 


\section{SOMMAIRE}

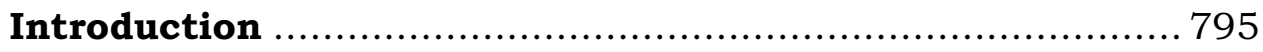

Partie 1 : Évolution historique du grief patronal.............. 798

Partie 2 : Quatre sentences arbitrales représentatives de la mouvance actuelle ............................................. 807

A. Paccar du Canada Ltée et TCA-Canada (griefs patronaux) [2007] ........................................ 807

B. Syndicat de l'enseignement de Lanaudière et

C. Commission scolaire des Samares (griefs patronaux) [2006] ........................................ 810

C. Transformateurs Pioneer ltée c. Métallurgistes unis d'Amérique, local 9414 [2007].....................8 811

D. Syndicat des technologues d'Hydro-Québec section locale 957 (SCFP/FTQ) c. Hydro-Québec [2006] ................................................ 816

Partie 3 : Quelques hypothèses/propositions quant au futur de cette pratique

Conclusion 825 


\section{Introduction}

Le rapport de travail salarié est depuis toujours générateur de tensions entre, d'une part, l'ambition de l'employeur qui cherche à optimiser la productivité de l'entreprise grâce à une main-d'œuvre efficace, et, d'autre part, les revendications des employés désireux de meilleures conditions de travail. Dans le contrat de travail classique, la force de travail du salarié est mise à la disposition de l'employeur, qui est ainsi justifié à donner des ordres à l'employé pour la bonne marche de l'entreprise. De ce rapport de subordination découle l'inégalité fondamentale du rapport salarial ${ }^{1}$. Dans ce contexte historique, l'avènement et le développement du syndicalisme ont en quelque sorte bridé l'arbitraire de l'employeur en milieu de travail en assumant la défense des intérêts de certains groupes de salariés par l'entremise de la négociation périodique des conditions de travail. L'encadrement juridique qui consacre la légitimité de l'action syndicale est aujourd'hui établi par le Code du travail ${ }^{2}$ québécois et, au niveau fédéral, par la partie I du Code canadien du travail ${ }^{3}$. Désormais, le syndicat majoritaire accrédité et l'employeur sont tenus de négocier de bonne foi une convention collective définissant les droits et obligations de chacun. L'institutionnalisation des rapports collectifs a ainsi permis aux syndicats accrédités d'imposer leurs vues auprès de certains employeurs, surtout ceux des PME, dont quelques-uns ont même dû déclarer faillite suite aux concessions exorbitantes faites aux syndiqués trop puissants ${ }^{4}$. Devant cette dissolution de l'autorité de la direction, on assiste peu à peu à l'émergence du phénomène relativement récent de grief patronal, déposé par l'employeur en vue de dénoncer le non-respect par la partie syndicale des dispositions de la convention collective.

1. Gregor MURRAY et Pierre VERGE, La représentation syndicale, Visage juridique actuel et futur, Saint-Nicolas, Les Presses de l'Université Laval, 1999, p. 1.

2. Code du travail, L.R.Q., c. C-27.

3. Code canadien du travail, L.R.C. 1985, c. L-2.

4. Jean BoIvin, "Règles du jeu et rapport de force dans les secteurs public et parapublic québécois ", (1979) 34 Rel. Ind. 3, 5. 
En effet, aux termes du Code du travail du Québec, un grief est " toute mésentente relative à l'interprétation ou à l'application d'une convention collective "5. Le droit au grief est donc dévolu à la fois aux parties syndicale et patronale. En particulier, le grief de l'employeur, tel que décrit par $\mathrm{M}$ e Fernand Morin et $\mathrm{M}^{\mathrm{e}}$ Rodrigue Blouin, " consiste en une réclamation par laquelle celui-ci soutient que le syndicat, voire même [sic] éventuellement un salarié, ne se conforme pas à une obligation de faire ou de ne pas faire que lui commande la convention collective et demande, en conséquence, un redressement " 6 . Comme dans le cas d'un grief syndical, l'essentiel du litige doit découler expressément ou implicitement d'une convention collective ${ }^{7}$. Les parties peuvent également élargir la notion légale de grief en exigeant expressément un recours à l'arbitre pour toute mésentente sur une condition de travail non prévue par la convention collective. Il s'agit des griefs dits assimilés par voie conventionnelle. En fonction de la finalité recherchée, on peut soit déposer un grief dit correctif pour réclamer compensation en raison d'une violation effective de la convention collective, soit loger un grief de principe pour rechercher une sentence

5. Code du travail, préc., note 2, art. $1 f$ ). En ce sens, le grief se distingue du " différend ", lequel est une mésentente survenant à l'occasion de l'élaboration même de la convention collective, soit au cours du processus de la négociation collective entre les parties (art. 1e) C.t.). Un différend peut également être soumis à l'arbitrage (art. 74 et suiv. C.t.). Ce processus est même obligatoire, à partir du moment où la négociation d'une première convention collective aboutit à une impasse (art. 93.1 et suiv. C.t.). Il revient alors à l'arbitre d'en déterminer le contenu (art. 93.4 C.t.).

6. Fernand MoRIn et Rodrigue Blouin, Droit de l'arbitrage de grief, 6e éd., Cowansville, Éditions Yvon Blais, 2012, p. 171, n III.38.

7. La compétence concomitante des tribunaux de droit commun a été définitivement rejetée au profit de l'exclusivité dans l'affaire St. Anne Nackawic Pulp \& Paper c. SCTP, [1986] 1 R.C.S. 704, par. 19 (ci-après "Nackawic "). Voir aussi Weber c. Ontario Hydro, [1995] 2 R.C.S. 929, par. 50 et suiv.; Nouveau-Brunswick c. O'Leary, [1995] 2 R.C.S. 967, par. 6. Dans le sillage de cette trilogie, l'arbitre dispose de tout litige qui naît d'une convention collective, à moins que la réclamation ne découle de l'insertion d'une clause discriminatoire dans une convention collective Québec (Commission des droits de la personne et des droits de la jeunesse) c. Québec (Procureur général), [2004] 2 R.C.S. 185. 
arbitrale à caractère déclaratoire confortant l'interprétation ou l'application de la convention collective avant que toute violation concrète ne soit intervenue, à condition que la mésentente entre les parties soit véritablement née et actuelle ${ }^{8}$. La plupart des conventions collectives prévoient aussi une procédure de règlement des griefs à l'interne plus ou moins détaillée impliquant le salarié et son représentant syndical d'un côté et le supérieur immédiat du salarié de l'autre. À défaut d'entente, le recours à l'arbitrage, prescrit à l'article 100 du Code du travail, est obligatoire et exclusif. L'arbitrage fait appel à l'intervention d'un tiers, neutre et distinct des deux parties contractantes. Contrairement au conciliateur et au médiateur, l'arbitre possède une fonction quasi judiciaire : en tant que tiers indépendant et impartial, il tranche les litiges opposant un syndicat à l'employeur à l'issue d'un débat contradictoire, et la décision de l'arbitre " est sans appel, lie les parties et, le cas échéant, tout salarié concerné "(art. 101 C.t.). Les tribunaux supérieurs en limitent par ailleurs la révision judiciaire conformément à l'article $139 \mathrm{du}$ Code du travail.

Qu'ils émanent du syndicat ou de la partie patronale, les griefs témoignent non seulement des problèmes éprouvés par les individus ou leurs représentants sur le site de travail, mais sont étroitement liés aux évolutions socio-économiques de la collectivité dans son ensemble. À cet égard, la tardive reconnaissance des griefs patronaux et leur prolifération dans les dernières années sont symptomatiques d'une réorientation de la dynamique sociale. Il serait ainsi opportun de s'interroger sur l'avenir du grief patronal (partie 3), et ce, à la lumière de son évolution historique (partie 1) et de l'orientation de la jurisprudence actuelle (partie 2).

8. Syndicat des professeurs de l'Université du Québec à Trois-Rivières c. Durand, C.A. Québec, $\mathrm{n}^{\circ}$ 200-09-000703-816, 5 février 1982, jj. Crête, Malouf et L'Heureux-Dubé, D.T.E. 82T-182 (C.A.). Plus récemment, voir Commission scolaire de St-Hyacinthe c. Syndicat canadien de la fonction publique, section locale 3259 (grief syndical), [2008] R.J.D.T. 800 (T.A.). 


\section{Partie 1 : Évolution historique du grief patronal}

La genèse du grief patronal est inextricablement liée à l'évolution du syndicalisme et de la convention collective. Au fil des ans, l'attitude de l'État face à l'action syndicale va " de la répression des principales manifestations de l'action syndicale à l'acceptation de leur légalité, puis même à la mise en place de régimes qui, avec une vue positive de l'action syndicale, lui confèrent une portée juridique, voire amplifient ses effets "9. À l'ère de la révolution industrielle, le droit applicable est largement influencé par l'idéologie dominante du libéralisme économique qu'incarne au Québec le Code civil du Bas-Canada ${ }^{10}$ : toute coalition ouvrière était perçue comme une conspiration illégale portant atteinte à la liberté de commerce. Une certaine reconnaissance légale des syndicats ouvriers ne s'est amorcée qu'à partir de 1872. Avec l'Acte concernant les associations ouvrières ${ }^{11}$, les syndicaux ouvriers bénéficient dès lors d'une immunité contre les poursuites (art. 2 et 3) et se sont vus conférer la faculté de conclure des conventions collectives (art. 4). Au Québec, la Loi des syndicats professionnels ${ }^{12}$ reconnaît la personnalité juridique aux associations patronales et syndicales qui le désirent (art. 1(4)) et légitime les conventions collectives qu'elles ont conclues (art. 5(9) et 15 à 19). Or, puisqu'aucune obligation de négocier n'est imposée à l'employeur, les moyens de pression comme la grève demeurent nécessaires pour sanctionner toute infraction au contenu des conventions. Il a fallu attendre la prospérité de l'après-guerre pour que la législation consente à des mesures plus favorables à l'action syndicale. Dans la perspective d'un réaménagement social, les autorités fédérales et québécoises, inspirées surtout des précédents américains $^{13}$, consacrent en 1944 les principes de la négociation obliga-

9. G. MurRay et P. Verge, préc., note 1, p. 9.

10. Marie-France BICH, "Droit du travail québécois : genèse et génération ", dans H. Patrick GLENN (dir.), Droit québécois et droit français : communauté, autonomie, concordance, Cowansville, Éditions Yvon Blais, 1993, p. 520 et suiv.

11. S.C. 1872 , c. 30 .

12. S.Q. 1924, c. 112.

13. National Labor Relations Act, (1935) 49 Stat. 449, c. 372 (“Wagner Act”). 
toire et de la représentation syndicale ${ }^{14}$. Le CP 1003 stipule que tout désaccord survenant durant la convention collective doit être résolu sans arrêt de travail. Pareille interdiction n'est acquise au Québec qu'en 196115. Auparavant, l'article 24(2) de la Loi des relations ouvrières suspend effectivement l'exercice du droit de grève au cours de la convention collective jusqu'à la soumission obligatoire d'un grief à l'arbitrage, mais les éventuelles décisions arbitrales ne liaient pas les parties, à moins qu'elles ne s'engageassent autrement à les respecter ${ }^{16}$. Depuis la réforme de 1961, le respect de la convention collective ne repose désormais plus uniquement sur la volonté des parties signataires, ni sur le recours aux moyens de pression; il relève d'un tiers, l'arbitre de griefs, doté d'un pouvoir décisionnel.

Cette première obligation imposée aux salariés syndiqués justifie à la fois la tardive apparition dans la jurisprudence des cas de grief déposé par l'employeur et le fait que ces premiers griefs patronaux se rapportent aux moyens de pression illégaux exercés par les salariés. D'après Luc Chamberland ${ }^{17}$, la première décision québécoise recensée faisant état d'un grief logé par l'employeur date de 1974. Dans cette affaire ${ }^{18}$, à la suite d'un arrêt illégal de travail de ses employés, la compagnie dépose un grief réclamant compensation au syndicat. Sa demande a été accueillie, et le syndicat a été condamné à payer $103000 \$$ à titre de dommagesintérêts. Le conseil d'arbitrage est d'avis que le syndicat a l'obligation de prendre le plus rapidement possible toutes les mesures positives adéquates afin de s'assurer du retour au travail

14. Arrêté en conseil 1003 du 17 février 1944 concernant les relations ouvrières en temps de guerre, (1944) 44 Gazette du travail 146; Loi des relations ouvrières, S.Q. 1944, c. 30.

15. Loi modifiant la Loi des relations ouvrières, S.Q. 1960 - 1961, c. 73, art. 6.

16. Fernand Morin, Jean-Yves BRIÈRE, Dominic RouX et Jean-Pierre VILlAGGI, Le droit de l'emploi au Québec, 4e éd., Montréal, Wilson \& Lafleur, 2010, p. $1291, \mathrm{n}^{\circ} \mathrm{IV}-176$.

17. Luc ChAmBerland, "Les griefs de l'employeur ", (1987) 47 R. du B. 822.

18. Les pâtes Domtar limitée, division Lebel-sur-Quévillon c. La Fraternité internationale des travailleurs de l'industrie des pâtes et papiers (492), [1974] R.D.T. 169. 
des employés, ce qui n'a pas été fait en l'espèce. Pour arriver à cette conclusion, le juge relève deux sentences arbitrales ontariennes des années $1950^{19}$ portant également sur des griefs patronaux en raison des grèves illégales exercées par les employés.

En 1948, s'est substituée au CP 1003 la Loi sur les relations industrielles et sur les enquêtes visant les différends du travail, à l'origine de la partie I du Code canadien du travail qui régit aujourd'hui les relations collectives du travail des entreprises relevant de la compétence fédérale 20 . L'actuel Code $d u$ travail québécois, sanctionné en 1964, représente quant à lui le prolongement historique de la loi de 1944. Au fil des décennies suivantes, autant le Parlement fédéral que la législature québécoise prescrivent des conditions minimales de travail dans les entreprises de leur ressort. L'on pense d'abord à la Loi sur les normes du travail21 de 1979. Également, la Loi sur la santé et la sécurité du travail22 protège la santé et l'intégrité physique du travailleur dans l'exercice de ses fonctions. Enfin, la Loi sur les accidents du travail et des maladies professionnelles ${ }^{23}$ vise la réadaptation du travailleur victime d'une lésion professionnelle en plus d'instituer un nouveau régime d'indemnisation fondé sur le risque.

Ainsi, quoique la définition textuelle du grief dans le Code $d u$ travail inclut depuis 1964 le grief patronal, l'orientation socialiste du droit du travail, souvent qualifié de droit ouvrier ou de droit du salariat, explique la lente reconnaissance des griefs émanant des employeurs qui se sentent lésés par les agissements excessifs de leur syndicat faisant fi de la convention collective dûment négociée. Jusqu'alors, les employeurs occu-

19. Canadian General Electric Co. Ltd., in re United Electrical, Radio and Machine Workers of America, Local 507, (1951) 2 L.A.C. 608; Polymer Corp. Ltd. and Oil, Chemical \& Atomic Workers, (1958) 10 L.A.C. 31.

20. Loi sur les relations industrielles et sur les enquêtes visant les différends du travail, S.R.C. 1952, c. 152, intégrée en 1970 au Code canadien du travail, L.R.C. 1985 , c. L-2.

21. L.Q. 1979, c. 45; aujourd'hui L.R.Q., c. N-1.1.

22. L.Q. 1979, c. 63; aujourd'hui L.R.Q., c. S-2.1.

23. L.Q. 1985, c. 6; aujourd'hui L.R.Q., c. A-3.001. 
paient une position privilégiée dans l'entreprise par leurs pouvoirs de direction, d'où l'élaboration d'un cadre législatif directement protecteur du salarié 24 . Depuis quand et dans quel contexte le pouvoir du syndicat militant a-t-il supplanté l'autorité traditionnelle de l'employeur dans les relations de travail?

Le premier cas québécois de grief patronal a été décidé en plein cœur de la crise économique des années 1970. L'effervescence syndicale était à son comble. Le taux de syndicalisation au Québec a passé de $27,1 \%$ en 1960 à $38 \%$ en $1975^{25}$. Les négociations stagnent : le recours au bluff et au jeu de la résistance "demeure presque dans tous les cas un élément inéluctable et nécessaire du jeu de la négociation "26. La multiplication des grèves dans tous les secteurs, désobéissant aux injonctions et défiant les lois spéciales ordonnant le retour au travail, perturbait sérieusement les services publics ${ }^{27}$. Dans ce contexte de la radicalisation de plusieurs demandes syndicales, l'employeur est devenu un contractant plus vulnérable susceptible d'être désavantagé en raison de la violation des dispositions de la convention collective par un syndicat vigoureux. En effet, la majorité des griefs déposés par les employeurs et rapportés dans les sentences arbitrales de l'époque portent sur la réclamation des dommages pour des arrêts de travail ou des piquetages illégaux ${ }^{28}$.

En 1980, la Cour suprême a eu l'occasion d'affirmer l'équivalence des griefs syndicaux et patronaux en se prononçant

24. Robert P. Gagnon, Louis LeBel et Pierre Verge, Droit du travail, 2e éd., Québec, Les Presses de 1'Université Laval, 1991, p. 29 et ss.

25. CALURA, 1987-1992, Rapport annuel du Ministre de l'Industrie, des Sciences et de la Technologie présenté sous l'emprise de la Loi sur les déclarations des personnes morales et des syndicats. Partie II - Syndicats, Ottawa, Statistique Canada, catalogue 71-202.

26. Gérard HÉBERT, "Les relations du travail au Québec : bilan des années 1970 ", (1981) 36 Rel. Ind. 715, 726.

27. Céline SAINT-PIERRE, "Aperçu historique du mouvement ouvrier au Québec : 1827-1976 ", (1978) 7-8 Politique aujourd'hui 109.

28. L. CHAMBERLAND, préc., note 17,822 . 
sur l'autorité de l'arbitre du grief dans l'affaire Shell29. Le pourvoi porte sur la compétence de l'arbitre à disposer d'une réclamation en dommages-intérêts contre les syndicats intimés à la suite d'une grève illégale prohibée par la convention collective. L'objection syndicale repose sur le fait que les réclamations en dommages intentées par l'employeur ne constitueraient pas des griefs au sens du Code du travail et de la convention collective. Sur la question de la compétence de l'arbitre, la Cour suprême renverse le courant jurisprudentiel de la Cour d'appel qui excluait de la juridiction du tribunal d'arbitrage toute violation d'une convention collective par un syndicat - plutôt que par un employeur - au motif qu'une violation ne saurait équivaloir à un grief en l'absence d'une difficulté véritable d'interprétation ou d'application de la convention collective. La majorité de la Cour d'appel semble en effet distinguer le cas sous étude de l'arrêt General Motors of Canada Ltd. c. Brunet30 :

Sur le plan de la mise en œuvre des recours, dans l'affaire Brunet c'est un salarié qui prétendait avoir des droits à faire valoir contre son employeur, et ce[,] essentiellement par application des stipulations de la convention collective. Dans le cas à l'étude, c'est un employeur qui prétend avoir subi des dommages-intérêts causés par des associations d'employés en conséquence d'une grève illégale ${ }^{31}$.

Infirmant cette opinion, le jugement de la Cour suprême est sans équivoque : toute violation d'une clause de convention collective, que ce soit par le syndicat ou l'employeur, constitue une mésentente relative à son application donnant ouverture au dépôt d'un grief de la compétence d'un arbitre. Sur ce point, le juge Chouinard s'exprime en ces termes :

Rien ne me paraît justifier cette distinction entre la réclamation d'un employeur et celle d'un employé. Il s'agit dans

29. Shell Canada Ltd. c. Travailleurs Unis du Pétrole du Canada, [1980] 2 R.C.S. 181.

30. [1977] 2 R.C.S. 537.

31. [1978] C.A. 404. 
chaque cas d'une réclamation monétaire découlant d'une violation d'une disposition de la convention collective et si l'une est de la compétence de l'arbitre, l'autre l'est aussi32.

Ce passage vient entériner la définition du grief énoncée dans le Code du travail, où aucune distinction n'a été faite entre les griefs d'un syndicat et ceux de la direction. Désormais, les catégories de griefs sont bel et bien considérées sur un pied d'égalité ${ }^{33}$. À la fin, le magistrat réserve deux questions connexes reformulées par le professeur Pierre Verge comme suit :

L'employeur, en raison de la prohibition législative de la grève durant la convention collective, aurait-il bénéficié au départ d'une option de recours en dommages-intérêts, l'un devant l'arbitre, fondé sur la prohibition conventionnelle, l'autre devant le tribunal civil, la Cour supérieure, basé celui-là sur la violation du Code du travail? D'autre part, quid dans le cas d'une convention collective qui, à la différence de la présente, ne contiendrait pas de disposition expresse réitérant en substance l'interdiction législative : y aurait-il absence de grief, partant de juridiction du tribunal d'arbitrage ${ }^{34}$ ?

Ces interrogations ont été résolues quelques années plus tard par la Cour suprême dans une affaire néo-brunswickoise mettant en cause un employeur qui se prétend - encore une fois préjudicié par une grève illégale de ses employés ${ }^{35}$. Cette grève

32. Shell Canada Ltd. c. Travailleurs Unis du Pétrole du Canada, préc., note 29, 185.

33. Cette position a été reprise quelques années plus tard dans l'affaire Purdel coopérative agro-alimentaire et Syndicat national des employés des produits laitiers du Bas St-Laurent, D.T.E. 84T-212 (T.A.), où l'arbitre Jean-Guy Michaud établit derechef que la définition du grief dans le Code $d u$ travail ne distingue pas entre les catégories de griefs et qu'une convention collective ne saurait limiter le droit de l'employeur d'en déposer pour réprimer la conduite répréhensible d'un syndicat récalcitrant.

34. Pierre VERGE, "L'octroi de dommages-intérêts par un tribunal d'arbitrage dans le cas d'une grève contraire à la convention collective ", (1980) 35 Rel. Ind. 578, 580.

35. Ste-Anne Nackawic Pulp \& Paper Co. Ltd. c. Section locale 219 du Syndicat canadien des travailleurs du papier, préc., note 7 . 
était interdite autant par la loi provinciale sur les relations de travail que par la convention collective. La Loi sur les relations industrielles du Nouveau-Brunswick ${ }^{36}$ prévoyait l'arbitrage obligatoire des griefs et édictait l'interdiction de déclencher une grève pendant la durée d'une convention. Après un examen de la valse-hésitation jurisprudentielle, le juge Estey, au nom de la majorité, a consacré la compétence exclusive de l'arbitre des griefs dans un litige résultant de la loi ou de la convention collective. Quant à la deuxième réserve exprimée dans l'affaire Shell, la juridiction du droit commun serait conservée en matière d'injonction :

Par ailleurs, lorsque la conduite de l'une ou l'autre partie à une convention collective dépasse le cadre de cette convention et porte atteinte à la disposition législative indépendante qui interdit la grève pendant la durée d'une convention collective, l'application complète du processus en matière de relations de travail établi par la législature est remise en question [...] La législature n'a donné aucune directive précise selon laquelle les tribunaux ne peuvent pas entendre de demande d'injonction lorsque tout le régime législatif en matière de relations de travail est bafoué, par exemple dans les affaires où les employés font la grève pendant la durée d'une convention collective ${ }^{37}$.

36. L.R.N.-B. 1973, c. I-4.

37. Ste-Anne Nackawic Pulp \& Paper Co. Ltd. c. Section locale 219 du Syndicat canadien des travailleurs du papier, préc, note 7 , par. 33. La compétence exclusive de l'arbitre des griefs s'étend, officiellement depuis le 15 juillet 2001 (Loi modifiant le Code du travail, instituant la Commission des relations $d u$ travail et modifiant d'autres dispositions législatives, L.Q. 2001, c. 26, art. 51), à l'émission d'ordonnances interlocutoires (pour une analyse de l'état du droit antérieur, voir Serge BRAULT et Maryse TREMBlay, "Le point sur le pouvoir de l'arbitre de griefs d'émettre des ordonnances interlocutoires ", (1995) 55 R. du B. 693). Le 1er avril 2011, la Cour d'appel du Québec a pour la première fois légitimé l'exercice exclusif de la juridiction arbitrale à l'émission d'une injonction permanente, considérée jusqu'alors comme un pouvoir inhérent de la Cour supérieure : Association des pompiers de Montréal inc. (APM) c. Montréal (Ville de), 2011 QCCA 631. 
La pratique du grief patronal serait également influencée par l'allongement de la durée des conventions collectives amorcé dans les années 1980, couplé à une précarisation du travail à l'échelle mondiale. Si l'incertitude par rapport à l'inflation et le contrôle des prix et des salaires de 1975 à 1978 justifiait le raccourcissement de la durée des conventions au Canada durant les années 1960 et 197038 , la récession économique débutée en 1982 et l'intensification de la concurrence dans les années 1990, conjuguées avec une faible inflation, sembleraient avoir inversé la tendance. Au Québec, la prédominance des conventions collectives de longue durée depuis les années 1990 serait en outre attribuable au déplafonnement, en 1994, de cette durée, jusqu'alors limitée à trois ans ${ }^{39}$. En effet, le pourcentage des conventions collectives de trente-sept (37) mois ou plus dans le secteur privé a augmenté de $20,8 \%$ en 1995 à $58,47 \%$ en 2011 , tandis que la durée moyenne des conventions est passée de 32,1 mois en 1994 à environ 48,90 mois en $2011^{40}$. Il devient dès lors nécessaire de pourvoir aux variations imprévues de l'économie et du coût de la vie dans les conventions collectives. On a ainsi observé, pour les années 1990 au Canada et au Québec, une augmentation simultanée de la fréquence des clauses de réouverture avec la durée des conventions collectives ${ }^{41}$. Malgré tout, les situations susceptibles d'irriter les

38. Louis CHRISTOFIDES, Les déterminants de la durée des conventions, Ottawa, Ministère du Travail du Canada, 1985.

39. Loi modifiant le Code du travail, L.Q. 1994, c. 6; Gérard HÉBERT, Traité de négociation collective, Boucherville, Gaëtan Morin Éditeur, 1992.

40. MinistĖRE DU TRAVAIL, Rapport sur les conventions collectives de longue durée de 1994 à 2002, Québec, Direction de la recherche et de l'évaluation, Québec, 2004, [Ressource électronique], en ligne: <http://www.travail.gouv.qc.ca/fileadmin/fichiers/Documents / conventi ons_collectives/RapportCCLD.pdf> (consulté le 14 juin 2011); MinistèrE DU TravaIL, "Tableau A-05 : Durée des conventions collectives ", Portrait statistique des conventions collectives analysées au Québec en 2011, Direction de l'information sur le travail, septembre 2012, en ligne: <http://www.travail.gouv.qc.ca/fileadmin/fichiers / Documents / conventi ons_collectives/Portrait_2011.pdf> (consulté le 8 avril 2013).

41. M. Lacroix, M. Hébert, N. Amyot, A. Charbonneau et T. Plante, Dispositions particulières des principales conventions collectives : conventions collectives de longue durée et réouverture des négociations, (1999) 2 Gazette du travail 42; Danièle MAYER et Reynald BOURQUe, "Le contenu des con- 
salariés au point de les amener à s'écarter des prescriptions de la convention collective deviennent plus nombreuses, puisqu'aucune obligation de renégocier les clauses - advenant un changement des circonstances - n'est imposée avant l'expiration de la convention. Cet état de fait pourrait contribuer à une certaine diversification des griefs patronaux constatée dans les années 1990. Les types de situation pouvant y donner ouverture sont notamment rapportés par Me Fernand Morin et Me Rodrigue Blouin ${ }^{42}$. Envers un salarié, l'employeur réclame le plus souvent un remboursement monétaire sur la base de la restitution de l'indu. Quant aux griefs dirigés contre le syndicat, ils portent principalement sur le remboursement d'engagements financiers - par exemple celui des salaires versés aux salariés libérés pour activités syndicales lorsque le syndicat s'était engagé à les remettre ${ }^{43}$ - ainsi que l'inexécution "d'une simple obligation de comportement qui contraint le syndicat ". L'on y retrouve entre autres les griefs en dommages pour des arrêts de travail ou ralentissements d'activités en contravention des stipulations de la convention collective ${ }^{44}$.

À l'aube du nouveau millénaire, quelles sont les tendances actuelles en matière de griefs patronaux? Pour mieux saisir l'orientation de la jurisprudence d'aujourd'hui, penchons-nous dès à présent sur quatre sentences arbitrales récentes s'y rapportant.

ventions collectives de longue durée au Québec de 1994 à 1996 ", dans C. BERNIER (dir.), Nouvelles formes d'emploi et diversification de la maind'œuvre, Québec, Actes du 36e congrès de l'Association canadienne de relations industrielles, p. 169.

42. F. MoRIN et R. Blouin, préc., note 6, p. $171-173$, n $^{\circ}$ III.38 et III.39.

43. Voir notamment Montréal (Communauté urbaine de) c. Chrétien, [1992] R.D.J. 298 (C.A.); Commission scolaire Cris c. Association de l'enseignement du Nouveau-Québec, [1990] R.D.J. 451 (C.A.).

44. À titre exemplatif: Cascade East-Angus inc. et Syndicat national des travailleurs de la pulpe et du papier de East-Angus inc., [1992] T.A. 307. 


\section{Partie 2 : Quatre sentences arbitrales représentatives de la mouvance actuelle}

Ces quatre sentences arbitrales seraient représentatives de l'état du droit quant aux griefs logés par l'employeur, autant en ce qui concerne les droits et obligations des deux parties que les nouveaux sujets qui peuvent en donner lieu comme des exigences procédurales. Les deux premiers cas, Paccar du Canada Ltée et TCA-Canada ainsi que Syndicat de l'enseignement de Lanaudière et Commission scolaire des Samares, confrontent les obligations du syndicat avec celles de l'employeur. La troisième affaire, Transformateurs Pioneer ltée c. Métallurgistes unis d'Amérique, local 9414, est un cas d'abus de procédure de grief que l'employeur reproche au syndicat, un recours bien établi dans la jurisprudence. La dernière sentence arbitrale, Syndicat des technologues d'HydroQuébec section locale 957 (SCFP/FTQ) c. Hydro-Québec, traite du délai de déchéance assez strict qui peut conduire au rejet d'une demande avant toute considération sur le fond du litige.

\section{A. Paccar du Canada Ltée et TCA-Canada (griefs patro- naux) [2007]}

Cette affaire 45 concerne deux griefs patronaux réclamant des dommages-intérêts au syndicat à la suite de deux arrêts de travail survenus pendant la durée de la convention collective. L'employeur est une entreprise de production de camions. La première interruption concertée de travail s'explique vraisemblablement par l'insatisfaction des employés quant à la qualité des produits de l'usine. Le second rassemblement non autorisé découlerait d'un incident fâcheux provoqué par l'arrogance d'un cadre.

Le Code du travail, à ses articles 107 et 108, prohibe tout arrêt de travail pendant la durée d'une convention collective, interdiction qui a été reprise à la clause 7.01 de la convention collective sous étude. Celle-ci explicite en outre les devoirs du syndicat

45. Paccar du Canada Ltée et TCA-Canada (griefs patronaux), [2007] R.J.D.T. 1752 (T.A.). 
en la matière, ce dernier s'engageant "à ne pas ordonner, encourager ni soutenir un ralentissement d'activités destiné à limiter la production ". À ce stade, l'arbitre Jean-Pierre Lussier a pris soin de préciser que nombre d'événements mécontentant les salariés sont susceptibles de se produire durant la vie d'une convention collective, mais, dans ces cas, "il faut tenter d'aplanir les difficultés par la négociation " et "il est clair que l'interruption concertée du travail est un moyen de pression proscrit par les parties " 46 . Examinant la jurisprudence arbitrale sur l'étendue des obligations du syndicat advenant un ralentissement d'activités destiné à limiter la production, l'arbitre retient deux sentences clés. La première, rendue par l'arbitre Huguette Gagnon 47, a statué que le syndicat ne peut se contenter de prouver qu'il n'en est pas à l'origine, mais doit également démontrer qu'il a fait tout son possible pour mettre fin à la grève, en signalant clairement son opposition, voire en menaçant les récalcitrants d'appliquer des sanctions disciplinaires. L'arbitre Jean-Pierre Lussier en conclut que les dirigeants syndicaux doivent y jouer un rôle proactif en posant des gestes concrets. La seconde décision, de l'arbitre Claude H. Foisy ${ }^{48}$, renchérit en spécifiant que cette obligation de syndicat en est une de diligence : celui-ci doit prendre tous les moyens raisonnables pour éviter ou mettre fin à un arrêt de travail illégal.

En l'occurrence, il n'a pas été prouvé que le syndicat a effectivement provoqué les deux ralentissements de travail. Or, les officiers syndicaux n'ont pas pris tous les moyens raisonnables pour les réprimer. Leur attitude était demeurée plutôt passive. Lors du premier arrêt de travail, au moins deux représentants syndicaux ont été vus au sein de l'attroupement. À l'arrêt de travail suivant, le président du syndicat, à l'instigation du directeur des ressources humaines, a demandé à deux reprises, sur un ton

46. Id., 1757.

47. Cascade (East Angus) inc. et Syndicat national des travailleurs de la pulpe et du papier de East Angus inc., préc., note 44.

48. Brasseries Molson et Union des routiers, brasseries, liqueurs douces et ouvriers de diverses industries, section locale 1999, [2002] $\mathrm{n}^{\circ} \mathrm{AZ}$ 02141212 , D.T.E. 2002T-775 (T.A.). 
neutre, aux travailleurs de reprendre le travail. Or, ce geste n'est pas suffisant aux yeux de l'arbitre. Si les circonstances varient dans chaque cas d'espèce, dans cette affaire, "il aurait au moins fallu que tous les officiers syndicaux se présentent ensemble avec Marc Tassé [le président du syndicat] devant les salariés et les invitent fermement, pour ne pas dire leur intiment l'ordre de retourner immédiatement à l'ouvrage en affirmant que ce n'était pas une façon de régler le problème relié à l'incident du 19 juillet "49. Le syndicat est donc responsable des dommages causés par ces arrêts de travail illégaux et tenu de compenser l'employeur pour le préjudice en résultant.

Deux points méritent d'être soulevés quant à la présente sentence. D'une part, elle s'inscrit dans le sillage du courant jurisprudentiel ${ }^{50}$ exigeant la responsabilisation du syndicat pour prévenir ou débloquer activement les grèves ou ralentissements d'activités illégaux. Les officiers syndicaux ne peuvent pas se contenter de rester dans la neutralité face aux agissements répréhensibles des salariés, mais doivent employer tous les moyens requis d'une personne raisonnable dans les circonstances pour montrer leur opposition et sanctionner les rebelles. D'autre part, l'employeur peut, au moyen d'un grief, chercher réparation de son préjudice uniquement à l'égard de son cocontractant, soit le syndicat, et non directement aux employés. Le syndicat pourra se retourner après coup contre les dirigeants syndicaux quant aux obligations qui leur incombent à ce titre. L'employeur ne pourrait

49. Paccar du Canada Ltée et TCA-Canada (griefs patronaux), préc., note 45, 1760.

50. Outre les causes citées dans la sentence, voir notamment: Syndicat des employés manuels de la Ville de Québec, section locale 1638 du Syndicat canadien de la fonction publique c. Ville de Québec, [1994] R.J.Q. 1552 (C.A.); Lawson Mardon Emballages inc., division de Montréal et Syndicat international des communications graphiques, section locale 555, [2001] R.J.D.T. 406 (T.A.); Syndicat canadien des communications, de l'énergie et du papier, section locale 720 et La Corporation Corbec, [2006] $\mathrm{n}^{\circ} \mathrm{AZ}$ 50387015, D.T.E. 2006T-753 (T.A.), requête en révision judiciaire rejetée; Réseau de transport de la Capitale c. Syndicat des salariées et salariés d'entretien du RTC, 2006 QCCA 706 (requête pour autorisation de pourvoi à la Cour suprême rejetée). 
que poursuivre un salarié pour une faute commise en contravention de ses devoirs de salarié.

\section{B. Syndicat de l'enseignement de Lanaudière et Commis- sion scolaire des Samares (griefs patronaux) [2006]}

Cette fois-ci51, les trente-neuf (39) griefs patronaux émanant d'une commission scolaire revendiquent le remboursement des sommes versées en trop à des enseignants à la suite d'une entente de redressement salarial intervenue entre le gouvernement du Québec et la Centrale de l'enseignement du Québec. Après avoir rejeté l'objection préliminaire de la prescription des recours, l'arbitre Maureen Flynn est d'avis qu'il s'agit bel et bien d'un cas de restitution de l'indu suite à une erreur de traitement informatique que la commission scolaire ne pouvait raisonnablement anticiper. La convention collective a pourvu aux mécanismes de restitution en cas de remise à un enseignant d'un trop payé à titre de rattrapage salarial. La commission doit, dans un premier temps, s'entendre avec l'enseignant concerné pour établir les conditions de remboursement et, à défaut d'une entente, la commission peut recourir à la compensation. Or, pour opérer compensation, les dettes doivent être certaines, liquides et exigibles. Dans la présente affaire, le syndicat a bel et bien signifié à la commission son refus de rembourser les versements excédentaires pour cause de prescription. Il s'agit donc d'une contestation soulevant l'interprétation et l'application de la convention collective qui rend les dettes incertaines. Dans ces circonstances, comme le relève Robert P. Gagnon, "la réclamation de l'employeur se heurt[ant] à une contestation du salarié sur la convention collective, elle deviendra l'objet d'un grief patronal soumis à la procédure d'arbitrage "52. D'ailleurs, en vertu de l'article 100.12b) du Code du travail, l'arbitre dispose d'un vaste pouvoir discrétionnaire - analogue à celui qui lui est conféré à l'article 1699 al. 2 du

51. Syndicat de l'enseignement de Lanaudière et Commission scolaire des Samares (griefs patronaux), [2006] R.J.D.T. 1225 (T.A.).

52. Id., 1236, citant Robert P. GAGNon, Le droit du travail du Québec, 6e éd., Cowansville, Éditions Yvon Blais, 2008, p. 620. 
Code civil du Québec en matière des restitutions en général - en ce qui a trait à la fixation des "modalités de remboursement d'une somme qu'un employeur a versée en trop à un salarié ". Le tribunal a ainsi réduit de moitié la restitution exigible d'une enseignante qui, sur la foi d'une assurance erronée, s'est privée des liquidités nécessaires au remboursement.

L'intérêt de cette cause est double. D'abord, en cas de restitution de l'indu dans un contexte d'interprétation et d'application de la convention collective, il n'est pas nécessaire pour l'employeur de loger un grief dans tous les cas. Si la dette du salarié remplit tous les critères exigés pour la compensation, soit la certitude, la liquidité et l'exigibilité ( $c f$. art. 1672 et suiv. C.c.Q.), l'employeur peut opérer compensation de sa propre initiative. Ensuite, en exerçant sa discrétion, l'arbitre tient à souligner que, dans certains cas, la négligence du payeur - le solvens -, en principe non pénalisante, peut être prise en compte par les tribunaux pour réduire, par souci d'équité, l'étendue de la restitution. En l'espèce, quoique la commission scolaire ait disposé de délais très serrés pour effectuer les premiers paiements de rattrapage salarial, elle a été négligente dans l'administration des appels des enseignants qui exprimaient des doutes quant à l'exactitude du paiement. Cette remarque est susceptible d'avoir quelques incidences sur le succès de ce type de griefs patronaux à l'avenir. Un employeur responsable doit minimiser ses dommages et ne pas adopter une conduite fautive au risque de se voir opposer une fin de nonrecevoir "[d]ans le cas où la conduite du solvens confine à l'incurie ou la négligence grossière ${ }^{53}$.

\section{Transformateurs Pioneer ltée c. Métallurgistes unis d'Amérique, local 9414 [2007]}

Dans cette affaire ${ }^{54}$, la compagnie Transformateurs Pioneer Ltée dépose un grief incident à la section locale des Métallurgistes

53. Id. 1237, citant Jean-Louis BAUdouIN et Pierre-Gabriel JoBIN, Les obligations, 6e éd., Cowansville, Éditions Yvon Blais, 2005, p. 560.

54. Transformateurs Pioneer ltée c. Métallurgistes unis d'Amérique, local 9414 , 2007 CanLII 12898 (QC S.A.T.). 
Unis d'Amérique pour abus de procédure de la part du syndicat. Ce dernier aurait demandé une remise d'audience injustifiée et tardive pour disposer du grief de suspension disciplinaire d'un employé. Celui-ci aurait refusé de collaborer avec le chef comptable de l'entreprise et l'aurait insulté devant d'autres personnes. L'employeur réclame le remboursement des frais engagés par la compagnie en raison de la conduite fautive du syndicat, notamment les honoraires et déboursés des procureurs ainsi que la totalité des frais de l'arbitre saisi du grief et des frais de consultant en ressources humaines. Le syndicat s'est par la suite désisté du grief initial.

À titre d'objection préliminaire, le syndicat a contesté la saisine du dossier patronal. Comme le grief principal a été retiré avant l'audience, le grief patronal incident ne saurait y survivre. De l'avis de l'arbitre Yvan Brodeur toutefois, cette objection n'est pas fondée. Si le grief de l'employeur est véritablement un grief incident étant donné que les faits pertinents sont intimement liés aux circonstances du grief initial, le grief incident ne devient pas pour autant l'accessoire du grief initial. À cet effet, l'article 172 al. $2 \mathrm{du}$ Code de procédure civile spécifie, in fine, que "[1]e tribunal reste saisi de la demande reconventionnelle, nonobstant un désistement de la demande principale ". Cette référence est éclairante même si le tribunal d'arbitrage n'est pas lié par ces dispositions. Ainsi, le désistement du grief syndical n'a aucunement affecté l'existence du grief incident.

Quant au fond, l'arbitre disserte sur la portée et les limites du devoir de représentation du syndicat vis-à-vis des salariés compris dans l'unité de négociation, tel qu'édicté à l'article 47.2 du Code du travail. Dès 1984, cette obligation a été circonscrite par la Cour suprême dans l'affaire La guilde de la marine marchande du Canada ${ }^{55}$ : si le droit de porter un grief à l'arbitrage est

55. La guilde de la marine marchande du Canada c. Gagnon et autre, [1984] 1 R.C.S. 509; voir aussi Diane VeILleux, "Le devoir de représentation syndicale : Cadre d'analyse des obligations sous-jacentes ", (1993) 48 Rel. Ind. 661. 
réservé au syndicat, le salarié n'a pas un droit absolu à l'arbitrage et le syndicat jouit d'une discrétion appréciable à cet égard, discrétion qui doit être exercée de bonne foi, de façon objective et honnête, après une étude sérieuse du grief et du dossier. Le syndicat doit soupeser les intérêts en présence avant d'agir; il doit d'un côté assumer ses obligations de juste représentation vis-à-vis du salarié et, de l'autre côté, agir de bonne foi à l'endroit de l'employeur, au risque de commettre un abus de droit à son égard. L'abus de droit, tel que défini à l'article 7 du Code civil du Québec, dépasse une simple erreur ou une négligence; il implique la mise en œuvre, de manière abusive, d'un droit dont l'exercice est normalement légitime. Dans les circonstances de l'espèce cependant, bien que l'attitude du syndicat ait incommodé l'employeur, il ne s'est pas comporté pour autant de façon excessive et déraisonnable. Le grief patronal a donc été rejeté.

L'abus de procédure de grief, invoqué par l'employeur comme demande reconventionnelle à un grief initial du syndicat, s'est multiplié ces dernières années ${ }^{56}$. Dans l'affaire Hôpital de Montréal pour enfants et Syndicat des technologues en radiologie du Québec ${ }^{57}$, l'arbitre Marc Boisvert a établi pour la première fois le caractère incident et accessoire du grief invoquant l'abus procédural. La réclamation en dommages par l'employeur ne constitue pas en soi un grief indépendant, puisqu'elle ne résulte pas d'une difficulté d'interprétation ou d'application de la convention collective à proprement parler. Le grief patronal pour abus de procédure

56. Voir notamment : Industries Leader inc. et Syndicat des salariées et sala-

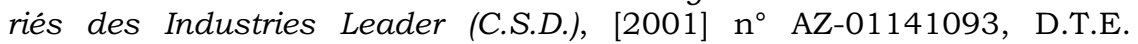
$2001 T-336$ (T.A.) (requête en révision judiciaire rejetée, [2001] $\mathrm{n}^{\circ} \mathrm{AZ}-$ 50099388, D.T.E. 2001T-884 (C.S.)); Aéroports de Montréal et Alliance de la fonction publique du Canada (unité des pompiers), [2003] $\mathrm{n}^{\circ} \mathrm{AZ}$ 03142120, D.T.E. 2003T-817 (T.A.); Autocar National Ltée et L'Union des employés et employées de service, section locale 800, [2004] $\mathrm{n}^{\circ} \mathrm{AZ}$ 50235448, D.T.E. 2004T-601 (T.A.); Hôpital général juif Sir Mortimer B. Davis et Syndicat des travailleuses et travailleurs de l'Hôpital général juif Sir Mortimer B. Davis/Jewish General Hospital (CSN) (grief patronal), [2007] $\mathrm{n}^{\circ}$ AZ-50429289, D.T.E. 2007T-474 (T.A.) (requête en révision judiciaire rejetée, C.S., 22-01-2008, 500-17-036435-074).

57. [1997] T.A. 1155. 
est plutôt assimilable à une demande reconventionnelle en matière civile. Cette approche est compatible avec les prescriptions $\mathrm{du}$ Code de procédure civile et de l'article 100.12 du Code du travail, ce dernier habilitant l'arbitre à rendre toute ordonnance propre à sauvegarder les droits de l'employeur et à imposer les dommages-intérêts appropriés, le cas échéant. Deux ans plus tard, cette position a été consacrée par la Cour d'appel dans l'affaire Hôpital général juif Sir Mortimer B. Davis c. Athanassiadis ${ }^{58}$. L'employeur avait saisi la Cour supérieure d'une action en dommages-intérêts réclamant le remboursement des frais liés à l'arbitrage, à la suite d'un grief syndical rejeté pour cause de prescription. Le juge Denis est d'opinion que la réclamation patronale relève de la convention collective. Lorsque l'employeur se rend compte en cours d'audience - ou avant - qu'il est victime d'une réclamation abusive ou farfelue, il peut, à ce moment précis ou dans le délai prévu à la convention collective, déposer un second grief, qui devient l'accessoire du premier et qui est disposé par le même arbitre, évitant de cette façon la multiplicité des recours. De surcroît, une clause conventionnelle prévoyant le paiement des frais d'arbitrage par l'employeur ne donnerait pas au syndicat une immunité pour en abuser et ne ferait pas obstacle à de telles réclamations incidentes intentées par l'employeur.

Considéré sous cet angle, l'apport de la sentence rendue dans l'affaire Transformateurs Pioneer consiste à préciser que le désistement du grief initial n'empêche pas la poursuite du grief incident. Quant aux obligations du syndicat, l'arbitre se réfère à la sentence rendue dans l'affaire Régie intermunicipale de récupération des Hautes-Laurentides et Syndicat des travailleuses et travailleurs de la Régie intermunicipale des Hautes-Laurentides (CSN)59, où l'arbitre François Hamelin a traité du devoir de représentation du syndicat envers les salariés. L'article $47.2 \mathrm{du}$ Code du travail accorde une discrétion appréciable au syndicat et ce n'est qu'en cas de décision arbitraire, capricieuse, discriminatoire ou abusive

58. C.A. Montréal, $\mathrm{n}^{\circ}$ 500-09-001558-949, 23 avril 1999, jj. Mailhot, Chamberland et Denis (ad hoc), D.T.E. 99T-462 (C.A.).

59. [2005] $\mathrm{n}^{\circ} \mathrm{AZ}-50310819$, D.T.E. 2005-533 (T.A.). 
que sa responsabilité est engagée60. En effet, si le salarié estime que l'association accréditée n'a pas exercé à son égard son devoir de représentation de manière juste et loyale, il peut porter plainte auprès de la Commission des relations du travail61. La gratuité et la facilité de ce recours ${ }^{62}$, jumelées à une incompréhension généralisée dans le milieu salarial quant à la nature et à l'intensité de l'obligation syndicale ${ }^{63}$, a rendu certains représentants syndicaux d'une prudence extrême, au point de décider de mener à terme des griefs non fondés pour acheter la paix ${ }^{64}$. Pour conclure que le syndicat devait se désister de son grief initial, il faut donc que l'employeur démontre que cette décision s'imposait absolument au syndicat, parce que celui-ci n'avait véritablement aucune chance de voir le grief accueilli, en tout ou en partie. Malgré ces réserves, un grief patronal invoquant l'abus de droit procédurier du syndicat est désormais ouvert aux employeurs.

60. Noël c. Société d'énergie de la Baie James, [2001] 2 R.C.S. 207, par. 48.

61. Code du travail, préc., note 2, art. 47.3 à 47.6.

62. Entre le 1er janvier 2006 et le 15 avril 2009, la Commission des relations du travail a reçu 1900 plaintes pour manquement au devoir de représentation, dont $63 \%$ concernaient des situations autres que des cas de renvoi ou de mesures disciplinaires. De toutes les plaintes reçues qui n'ont pas fait l'objet d'un désistement ou d'un règlement, $86 \%$ sont rejetées par la Commission : Nancy MARTEl et Pierre E. MOREAU, Le devoir de juste représentation, Markham (Ontario), Lexis Nexis Canada, 2009.

63. Anne PinEAU, "Le devoir de représentation syndicale : une dérive inquiétante ", dans Développements récents en droit du travail, Service de la formation continue du Barreau du Québec, 2008, Droit civil en ligne (DCL), EYB2008DEV1486.

64. Id. En effet, un manquement au devoir de juste représentation emporte de lourdes conséquences pour le syndicat: celui-ci s'expose à une plainte portée à l'initiative du salarié lésé qui a subi un renvoi ou une mesure disciplinaire, ou qui croit avoir été victime de harcèlement psychologique pour forcer le renvoi de sa réclamation à l'arbitrage (art. 47.3 C.t.). Depuis le 1 er janvier 2004, la compétence exclusive de la CRT a été élargie pour disposer de tout recours exercé par un salarié à l'encontre de son syndicat relatifs à la négociation, à l'application et à l'interprétation de la convention collective (art. 114 C.t.). Outre une condamnation en dommages, la CRT peut même à cette occasion ordonner au syndicat de rembourser au salarié les honoraires extrajudiciaires encourus pour faire valoir ses droits devant la Commission (Métallurgistes unis d'Amérique, section locale 9414 c. Castonguay, 2007 QCCA 1766). 


\section{Syndicat des technologues d'Hydro-Québec section lo- cale 957 (SCFP/FTQ) c. Hydro-Québec [2006]}

Cette affaire 65 se rapporte à un grief patronal déposé par Hydro-Québec réclamant à un de ses employés le remboursement des frais de repas et de déplacement. Ce litige s'est buté contre l'objection préliminaire du syndicat soulevant la prescription du recours. Le grief patronal, qui provient des irrégularités sur les comptes de dépenses de l'employé en 1999, a été déposé le 6 mai 2003, puis soumis à l'arbitrage le 16 juin 2003.

À défaut d'un délai spécifiquement prévu dans la convention collective pour le dépôt d'un grief patronal, est-ce la prescription de six mois prévue à l'article 71 du Code du travail qui s'applique, ou est-ce plutôt la prescription triennale du droit commun? Sur cette interrogation, l'arbitre Diane Sabourin rappelle dans un premier temps qu'initialement l'article $71 \mathrm{du}$ Code du travail a été interprété comme ayant un caractère supplétif et ne s'applique par conséquent que lorsque la procédure de griefs et d'arbitrage prévue dans une convention collective est silencieuse quant au délai. Toutefois, deux raisons principales plaident pour la position contraire. D'un côté, les droits et recours que l'employeur entend exercer " naissent d'une convention collective ", comme le stipule le Code du travail, et non en vertu des dispositions du Code civil. En effet, toutes les sommes réclamées par l'employeur à son salarié ont été versées en fonction des dispositions de la convention collective. De l'autre côté, il y a lieu de considérer sur un pied d'égalité les droits et recours prévus pour chacune des deux parties à la convention collective. Une réclamation ne saurait constituer une action civile - plutôt que l'arbitrage de griefs - uniquement parce qu'elle émane d'un employeur au lieu d'un syndicat. Par ailleurs, le libellé de l'article 2925 du Code civil indique clairement qu'il ne s'applique qu'aux cas où "le délai de prescription n'est pas autrement fixé ". Ainsi, le délai de prescription de 6 mois prévaut et court à compter de la naissance de la

65. Syndicat des technologues d'Hydro-Québec section locale 957 (SCFP/FTQ) c. Hydro-Québec, 2006 CanLII 19306 (QC S.A.T.). 
dette de l'employé, "à compter du jour où la cause de l'action a pris naissance ". Le point de départ de la prescription étant la date 15 janvier 2001, le recours est d'ores et déjà prescrit.

Il est vrai que l'approche adoptée généralement par les arbitres et consacrée par la Cour suprême n'est pas très exigeante quant au respect des procédures. En matière d'arbitrage des griefs, le fond l'emporte sur la forme, suivant les termes de la Cour suprême dans l'affaire Parry Sound ${ }^{66}$ :

En règle générale, il est évidemment important que les parties à une convention collective se conforment aux exigences procédurales qui y sont prévues (...) Cela dit, il importe de reconnaître le consensus général chez les arbitres que, dans la mesure du possible, un grief ne devrait pas être gagné ni perdu pour un vice de forme, mais plutôt en raison de son bien-fondé.

Ces principes, énoncés dans le cas d'un grief syndical, sont tout à fait transposables aux griefs patronaux. De surcroît, l'article 100.2.1 du Code du travail prévoit qu' "[a]ucun grief ne peut être rejeté pour vice de forme ou irrégularité de procédure ". À l'interne, les parties sont libres d'adopter la procédure de leur choix, sous réserve de l'arbitrage final obligatoire au terme du processus. Lorsqu'aucune procédure interne n'est prévue dans la convention collective, le grief de l'employeur est soumis directement à l'arbitrage. Or, faut-il obligatoirement respecter les étapes internes de règlement des griefs avant qu'un grief soit ultimement porté à l'arbitrage? La réponse varie suivant les termes de la convention collective. Si les parties ont exprimé leur intention arrêtée de suivre à la lettre les modalités établies dans la convention, elles ne pourront revenir sur leur position et l'arbitre ne pourra que sanctionner le manquement procédural ${ }^{67}$. Par contre, quand la

66. Parry Sound (district), Conseil d'administration des services sociaux c. S.E.E.F.P.Q., section locale 324, [2003] 2 R.C.S. 157, par. 68.

67. F. Morin et R. Blouin, préc., note 6, p. $327-332$; Robert P. GAGNon, Le droit du travail au Québec, 6e éd., Cowansville, Éditions Yvon Blais, 2008, p. $590-592$. 
procédure n'est pas de déchéance et que l'irrégularité n'a occasionné aucun préjudice à l'autre partie, le grief doit être tranché sur le fond, et non rejeté en raison de simples accidents de parcours. Le dépôt du grief n'est soumis à aucun formalisme : une lettre adressée au syndicat faisant état de l'objet du grief et du correctif recherché a été jugée suffisante ${ }^{68}$.

En fait, la seule brèche à cette trame procédurale plutôt élastique réside dans la question de la prescription. Les délais de rigueur ${ }^{69}$ prévus dans les conventions collectives en ce qui concerne le dépôt des griefs ou leur renvoi éventuel à l'arbitrage s'avèrent généralement courts, à telles enseignes que le législateur ait cru nécessaire d'intervenir pour en fixer un minimum à quinze jours de la naissance de la cause d'action ${ }^{70}$. La prescription statutaire supplétive est de six mois (art. 71 C.t.). Le seul et unique cas où il est permis de déférer hors délai un grief à l'arbitrage se produit lorsqu'une des parties refuse de se conformer à un règlement négocié au préalable (art. 100.0.2 C.t.). Vu qu'un bon nombre de griefs patronaux ont été rejetés sur la base de telles objections ${ }^{71}$, les employeurs et les syndicats doivent agir avec célérité pour faire préserver leur droit.

Fort de ces différents constats, l'on pourrait formuler quelques hypothèses sur l'avenir des griefs patronaux. Telle qu'analysée dans les pages qui suivent, l'évolution des relations

68. S.P.B. Canada Inc. c. Union des employés des cartonniers standard, [1985] T.A. 273.

69. Voir Syndicat des employés du CUS McGill (FAS-CSN) c. CUSM - Hôpital général de Montréal, 2009 CanLII 76321 (QC S.A.T.).

70. Art. 100.0.1 C.t. : "Un grief soumis à l'autre partie dans les quinze jours de la date où la cause d'action a pris naissance ne peut être rejeté par l'arbitre au seul motif que le délai prévu à la convention collective n'a pas été respecté."

71. Voir notamment: Québec (Ville de) c. Association des pompiers professionnels de Québec, 2001 CanLII 7615 (QC S.A.T.); Commission Scolaire Crie c. Syndicat des professionnelles et professionnels en milieu scolaire du Nord-Ouest, 2003 CanLII 16044 (QC S.A.T.); Association des enseignantes et enseignants de Montréal c. Picher, 2006 QCCS 1715; Commission scolaire de la Pointe-de-l'̂lle c. Dulude, 2007 QCCS 352 
syndicales patronales serait éminemment tributaire du devenir socioéconomique de nos collectivités au sens large.

\section{Partie 3 : Quelques hypothèses/propositions quant au futur de cette pratique}

Première impression : en ce qui concerne l'objet des griefs patronaux, l'employeur aurait de plus en plus d'occasions d'invoquer l'abus de la procédure de grief à l'encontre du syndicat à titre de demande reconventionnelle.

À mesure que les conventions collectives s'allongent et se complexifient, cherchant à réglementer la vie au travail dans ses moindres détails, davantage d'obligations incombent désormais à l'employeur, que ce soit au chapitre des avantages sociaux, de la sécurité syndicale ou des mesures de protection du revenu. Au cours des vingt dernières années, y ont aussi été ajoutées des clauses abordant des thèmes moins traditionnels comme les droits des salariés en matière de formation professionnelle et la conciliation travail-vie personnelle. À titre indicatif, près de $60 \%$ des conventions collectives $\mathrm{du}$ secteur privé déposées en 2008 comportaient au moins une clause portant sur la formation, le recyclage ou le perfectionnement. De plus en plus de conventions collectives incluent en effet des dispositions de cette nature; des $38,9 \%$ qui en traitent en 1988-1991, le pourcentage a grimpé à $69,4 \%$ en 2006-200972. Dans le même ordre d'idées, la proportion du coût des avantages sociaux dans le secteur privé syndiqué du Québec, que ce soit le temps chômé payé, les régimes privés de retraite et d'assurances collectives, les régimes publics ou autres avantages, varie d'environ $36 \%$ à près de $43 \%$ des coûts salariaux selon les catégories d'emploi ${ }^{73}$. De plus, la proportion des conven-

72. Pour les conventions de 1988-1991 : CENTRE DE RECHERCHE ET DE STATISTIQUES SUR LE MARCHÉ DU TRAVAIL (diverses années), Conditions de travail contenues dans les conventions collectives au Québec. Pour les conventions de 2006-2009: Système d'information "Gestion des relations du travail ", ministère du Travail, 31 décembre 2009.

73. Patrice JaletTe et Gilles TRUdeAu (dir.), La convention collective au Québec, 2e éd., Montréal, Gaëtan Morin éditeur, 2011, p. 337. 
tions collectives prévoyant des dispositions sur le congé parental a plus que quadruplé dans les périodes de référence 1988-1991 et 2003-200674. Aussi, près de 28\% des mères québécoises reçoivent des prestations complémentaires de leur employeur en sus des prestations du Régime québécois d'assurance parentale. Ces prestations complémentaires sont versées près de quatre fois plus fréquemment en milieu syndiqué qu'en milieu non syndiqué75.

Toutes ces exigences variées pourraient donner aux salariés ou au syndicat de multiples occasions de contester les décisions de l'employeur qui s'écarteraient des prescriptions de la convention. Entre les années 2000 et 2003, l'objet de ces griefs concerne le plus souvent les questions disciplinaires tel le congédiement ou la suspension ainsi que des questions de rémunération (indexation des salaires, primes, heures supplémentaires et assurancesalariale), la réclamation de poste ou les diverses modifications aux conditions de travail 76 . De surcroît, l'accumulation des clauses dans les conventions collectives est susceptible d'amener beaucoup de lourdeur dans l'application et bien des difficultés d'interprétation. Considérés sous cet angle, les griefs syndicaux déposés en vue de dénoncer la violation ou l'apparente violation de la convention par la partie patronale risquent de devenir plus nombreux, d'où l'intérêt d'un grief incident lorsque le grief principal se révèle manifestement mal fondé ou superflu.

Deuxième constat : les griefs patronaux contestant plus spécifiquement les arrêts ou ralentissements de travail illégaux pourraient se maintenir au cours des prochaines années. Certes, les conflits de travail - constitués majoritairement de grèves - ont diminué de près de moitié depuis 2001. S'en trouve corrélativement réduit le nombre de travailleurs touchés, lequel a passé de

\footnotetext{
74. Id., p. 374.

75. Katherine MARSHALL, "Prestations supplémentaires versées par l'employeur ", Perspective, février 2010, Ottawa, Statistique Canada, p. 5 $-14$.

76. Lyne JonCAS, Le système québécois d'analyse des sentences arbitrales de grief : un aperçu, Ministère du Travail, 2004, p. 10.
} 
52597 en 2001 à 11281 en 200977 . Cependant, la durée des conventions collectives s'est allongée de façon substantielle à la suite de son déplafonnement législatif intervenu en 1994, alors que les clauses de réouverture permettant à l'une des parties de demander une renégociation avant l'expiration de la convention collective demeurent rares. En fait, seulement $2 \%$ des conventions collectives entre 2003 et 2007 comportent de telles clauses 78 . Pour les employeurs, une convention collective de longue durée est synonyme de stabilité et de certitude, les conditions qui y sont stipulées ne sont plus sujettes aux fluctuations économiques. Elle permet également de réduire les coûts associés aux négociations collectives et aux conflits de travail. Pour les syndicats toutefois, l'accroissement de cette durée suspend leur droit de négocier de meilleures conditions de travail en vue de rétablir l'équilibre rompu par des circonstances nouvelles imprévisibles, le cas échéant. Ces intérêts opposés peuvent éventuellement donner lieu à des frictions entre les parties syndicale et patronale, conduisant à l'usage des moyens de pression illégaux lorsque chacune des parties reste campée sur sa position malgré les aléas de l'économie. À cet égard, l'enjeu salarial demeure un point des plus conflictuels puisque le niveau des salaires est une des conditions les plus intimement liées aux pressions économiques et à la situation financière de l'entreprise. En fait, $44 \%$ des conflits de travail survenus durant la période 2004-2008 sont provoqués par le désaccord des parties sur cette question 79. Alors que l'employeur veut contenir les hausses salariales pour optimiser la productivité de l'entreprise et supporter la concurrence, les revendications salariales cherchent plutôt à les augmenter au profit des salariés.

Troisième hypothèse : il serait à prévoir que les entreprises seront davantage axées sur la gestion du grief à l'interne et qu'elles gagneraient à recourir à une médiation pré-arbitrale avant de se lancer sur le forum arbitral comme tel. Malgré une augmen-

77. InstituT DE LA STATISTIQUe DU QUÉBEC, Annuaire québécois des statistiques du travail, Portrait des principaux indicateurs des conditions et de la dynamique du travail, vol. 6 no. 2, 2010, p. 219 et 220.

78. P. JaletTe et G. TRUdeau (dir.), préc., note 73, p. 53.

79. Id., p. 439. 
tation générale du nombre de griefs d'année en année, soit près de mille entre les années 2000 et 2003, plus de la moitié des griefs déposés ont été réglés à l'amiable. Excluant ce pourcentage élevé de règlements hors cour, la majorité des griefs - soit en moyenne $65 \%$ - ont été rejetés par l'arbitre ${ }^{80}$. En même temps, il y a eu, au cours de la décennie, une diminution draconienne de près de $40 \%$ du nombre de griefs - toute catégorie confondue - ayant fait l'objet d'une sentence arbitrale, passant de 2591 décisions recensées en 1992-1993 à 1503 décisions rendues en 2004-200581.

En effet, la procédure de règlement des griefs ne comporte pas que des avantages. Certes, l'arbitrage se veut avant tout une alternative pacifique de résolution des conflits qui offre aux salariés de meilleures possibilités de participer à l'élaboration des solutions, au lieu de recourir à des moyens radicaux comme la grève ou le lock-out. C'est d'ailleurs cette considération qui a incité le législateur à instituer l'arbitrage obligatoire au début des années 1960 pour faire respecter les dispositions des conventions collectives. Cependant, la procédure de règlement des griefs est parfois perçue comme "une procédure inflexible, laborieuse et dilatoire "82. Le délai moyen pour rendre une décision arbitrale, de la nomination de l'arbitre au prononcé de la décision, a presque doublé de 1990 à 2010, passant de 259 jours à 470 jours ${ }^{83}$. Si les parties sont largement responsables de cette lenteur, n'empêche que tout le processus est en soi coûteux en temps et en ressources : outre les honoraires d'arbitre et des assesseurs, les déboursés des procureurs et des témoins experts, dont la présence risque par ailleurs d'augmenter considérablement les délais en

80. L. JONCAS, préc., note 76 , p. 5 .

81. COMITÉ DE TRAVAIL DE L'ORDRE DES CONSEILLERS EN RESSOURCES HUMAINES ET EN RELATIONS INDUSTRIELLES AGRÉÉS DU QUÉBEC, Relations du travail au Québec, Bilan et perspectives d'avenir, avril 2005, en ligne: $<$ http://www.eri.umontreal.ca/personnel/Jalette_Patrice/bilan.pdf > (consulté le 13 juin 2011).

82. Jean-Claude BERNATCHEZ, La convention collective, savoir la négocier, l'interpréter, l'appliquer, Québec, Les Presses de l'Université du Québec, 2003, p. 304.

83. CONSEIL CONSUlTATIF DU TRAVAIL ET DE LA MAIN-D'OEUVRE (CCTM), Liste annotée d'arbitres de grief, 2010-2011, Québec, Ministère du Travail, p. 12. 
raison de leur disponibilité réduite, les coûts de libération pour les employés et des témoins sont autant des éléments démotivants, et ce, sans discuter de leurs impacts sur la productivité de l'entreprise.

À l'opposé, la procédure interne de règlement des griefs se démarque par son accessibilité, en mettant en présence les personnes immédiatement impliquées. L'efficacité de ce mécanisme est démontrée par le fait que plus de $95 \%$ des griefs sont réglés par les parties elles-mêmes, sans l'intervention d'un tiers ${ }^{84}$. Aussi, la médiation pré-arbitrale de griefs pourrait être appelée à jouer un rôle clé, en ce que, sans se substituer à la procédure d'arbitrage en tant que telle, cette démarche volontaire permet aux parties de bénéficier des conseils et de l'assistance d'un tiers neutre aux conflits pour aboutir à un règlement satisfaisant des griefs avant qu'ils ne soient portés à l'arbitrage. Ainsi, l'intervention d'une partie neutre qui n'a d'autres intérêts que de voir les griefs classés éclairera la vision dans laquelle chacune des parties est enfermée, et sa présence pourrait modifier l'attitude de chacune des parties qui, se sentant observée, aurait tendance à s'investir davantage dans une analyse plus poussée du dossier à traiter 85 .

Dernière observation : dans une perspective globale, les griefs patronaux demeureraient un phénomène marginal. D'une part, le taux de présence syndicale, ce pourcentage de personnes assujetties à une convention collective, s'est stabilisé au cours de la décennie, malgré une légère régression. En 2010, 39,6\% des salariés québécois étaient assujettis à une convention collective de travail, comparé à un taux de 40\% l'année précédente et au taux moyen de 40,3\% observé au Québec pour la période 2001-2010. Ce pourcentage masque pourtant l'écart flagrant entre le secteur

84. G. HÉBERT, Traité de négociation collective, préc., note 39, p. 204.

85. Pour une étude de cas en milieu hospitalier québécois, voir Véronique ROBERGE, L'impact de la médiation pré-arbitrale des griefs : une étude de cas en milieu hospitalier québécois, mémoire de maîtrise, Montréal, Faculté des études supérieures (relations industrielles), Université de Montréal, 1995. 
privé et le secteur public. Si le secteur privé regroupe les trois quarts des salariés, son taux de présence syndicale a diminué de $27,9 \%$ à $25,3 \%$ depuis 2001 , comparativement à une augmentation de $81,3 \%$ à $82,2 \%$ dans le secteur public 86 . D'autre part, le cadre législatif des rapports collectifs de travail s'est davantage orienté vers la déréglementation au cours des trente dernières années, rendant de plus en plus difficiles la syndicalisation des travailleurs et la négociation collective. Cette volonté gouvernementale s'inscrit dans la mouvance de l'idéologie néolibérale en vogue dans les politiques publiques depuis les années 1970 et 1980 prônant la privatisation, la dérégulation du marché du travail, le retrait de l'État et le soutien aux investissements privés ${ }^{87}$. L'abolition de la limite maximale à la durée des conventions collectives, la modification de l'article $45 \mathrm{du}$ Code du travail - facilitant le recours à la sous-traitance dans les milieux syndiqués et l'exclusion de certains travailleurs de l'application des décrets de convention collective en constituent des exemples flagrants. Aussi, un cadre législatif de plus en plus précis, prescrivant avec minutie les conditions minimales de travail, risquent d'amenuiser le rôle de la convention collective dans la régulation des conditions de travail. Référons-nous à la Loi sur l'équité salariale ${ }^{88}$, à la Loi favorisant le développement et la reconnaissance des compétences de la main-d'œuvre 89 et au renouvellement de la Loi sur les normes $d u$ travail. Tous ces facteurs ne plaident pas pour une croissance importante du taux de couverture conventionnelle et des effectifs syndicaux ${ }^{90}$. Or, pas de syndicat, pas de convention et partant, plus de grief.

86. Alexis Labrosse, La présence syndicale au Québec en 2010, Québec, Ministère du Travail, 2011.

87. Sophie FONTAINE-BÉGIN, Influence du néolibéralisme de l'État sur les stratégies de l'acteur syndical dans le secteur public québécois : l'étude du cas de la FIIQ, mémoire de maîtrise, Montréal, École de relations industrielles, Université de Montréal, 2007.

88. L.R.Q., c. E-12.001.

89. L.R.Q., c. D-8.3.

90. P. Jalette et G. Trudeau (dir.), préc., note 73, p. 53. 


\section{Conclusion}

La convention collective vise à préserver les droits des salariés qui y sont assujettis contre l'autorité intrusive de l'employeur, qui, au sein de l'entreprise, occupe le haut du pavé. Résultat d'une négociation laborieuse entre le syndicat et la direction, elle est avant tout l'engagement de l'employeur à respecter ses propres obligations en reconnaissant les concessions faites aux employés, depuis les bénéfices traditionnels comme la sécurité syndicale, l'ancienneté, les mesures disciplinaires et les avantages sociaux jusqu'aux revendications plus innovatrices comme la formation continue et la conciliation travail-vie personnelle. En raison de sa nature même, une convention collective impose plus d'obligations pour l'employeur que les salariés assujettis, d'où la fréquence beaucoup plus élevée des griefs syndicaux dénonçant la violation par l'employeur d'une ou de plusieurs stipulations, notamment en ce qui concerne les questions de rémunération ou de mesures disciplinaires jugées déraisonnables.

Cette dynamique fondamentale des rapports collectifs limite grandement les causes d'action des griefs patronaux. La négociation, puis la conclusion d'une convention collective, emporte inévitablement une dissolution, plus ou moins prononcée, du pouvoir patronal dans bien des domaines qui relèveraient autrement et normalement de son ressort exclusif. À l'opposé, qu'est-ce que l'employeur pourrait trouver à redire contre un syndicat ou ses salariés? Visiblement, l'éventail des motifs d'insatisfaction se rétrécit, si ce n'est l'exercice inadéquat d'activités syndicales qui risque de causer du tort à l'employeur et de troubler le climat général de travail. Après tout, en contrepartie des gains syndicaux, la première obligation imposée au syndicat est bel et bien l'interdiction de perturber les activités de l'entreprise pendant la durée d'une convention collective en recourant à des moyens de pression préjudiciables à l'employeur. La violation de cet engagement, surtout dans les périodes de ralentissement économique, explique la genèse des premiers griefs patronaux. 
Depuis, les cas de ces griefs se diversifient avec la complexité croissante des conventions collectives, dictant à l'une et à l'autre partie de nouveaux engagements. Outre les actions en dommages à la suite d'arrêts de travail illégaux, l'on recense les réclamations de l'employeur exigeant le remboursement des salaires versés pour activités syndicales ou de salaires par erreur, lorsque les salariés abusent, sciemment ou par mégarde, des avantages concédés dans les conventions collectives. Puis, lassés des griefs syndicaux contestant l'application légitime des mesures disciplinaires, dont le recours à l'arbitrage est souvent lourd de conséquences pécuniaires, les employeurs répliquent de plus en plus fréquemment en invoquant, comme demande reconventionnelle au grief principal, l'abus de procédure du syndicat dans le dépôt des griefs frivoles qui n'ont aucune chance raisonnable d'être accueillis. Cette tendance découlerait quelquefois d'un exercice parfois "excessif " du devoir de représentation du syndicat vis-à-vis des salariés compris dans l'unité de négociation, tel qu'édicté à l'article 47.2 du Code du travail et circonscrit par la jurisprudence de la Cour suprême du Canada, alors que la "banalisation " du contrôle de l'action syndicale pourrait inciter certains syndicats du secteur privé à renvoyer à l'arbitrage nombre de griefs de leurs salariés à l'encontre de l'employeur, au risque de commettre un abus de droit à son égard.

En définitive, l'on pourrait raisonnablement inférer que les sentences arbitrales disposant des griefs patronaux demeureront rarissimes dans un avenir rapproché. Les causes d'action, qu'elles soient légales ou conventionnelles, s'y révèlent de prime abord beaucoup moindre que les motifs de reproche à l'égard d'un employeur qui outrepasserait les limites tracées par les conventions collectives quant à l'exercice de ses droits de direction. Malgré la compétence exclusive de l'arbitre en la matière et l'efficacité de ce forum en tant qu'institution, la baisse du taux de syndicalisation ne favoriserait vraisemblablement pas une prolifération numérique des griefs patronaux. 